UCRL-JC-130496

PREPRINT

\title{
Electromagnetic Interactions GEneRalized (EIGER): Algorithm Abstraction and HPC Implementation
}

\author{
R. M. Sharpe, J. B. Grant, and N. J. Champagne (LLNL) \\ D.R. Wilton and D.R. Jackson (University of Houston) \\ W.A. Johnson and R. E. Jorgensen (Sandia National Labs) \\ J. W. Rockway and C.W. Manry (SPARWAR)
}

This paper was prepared for submittal to the

29th Plasma Dynamics and Lasers Conference

Albuquerque, NM

June 15 - June 18,1998

April 21, 1998

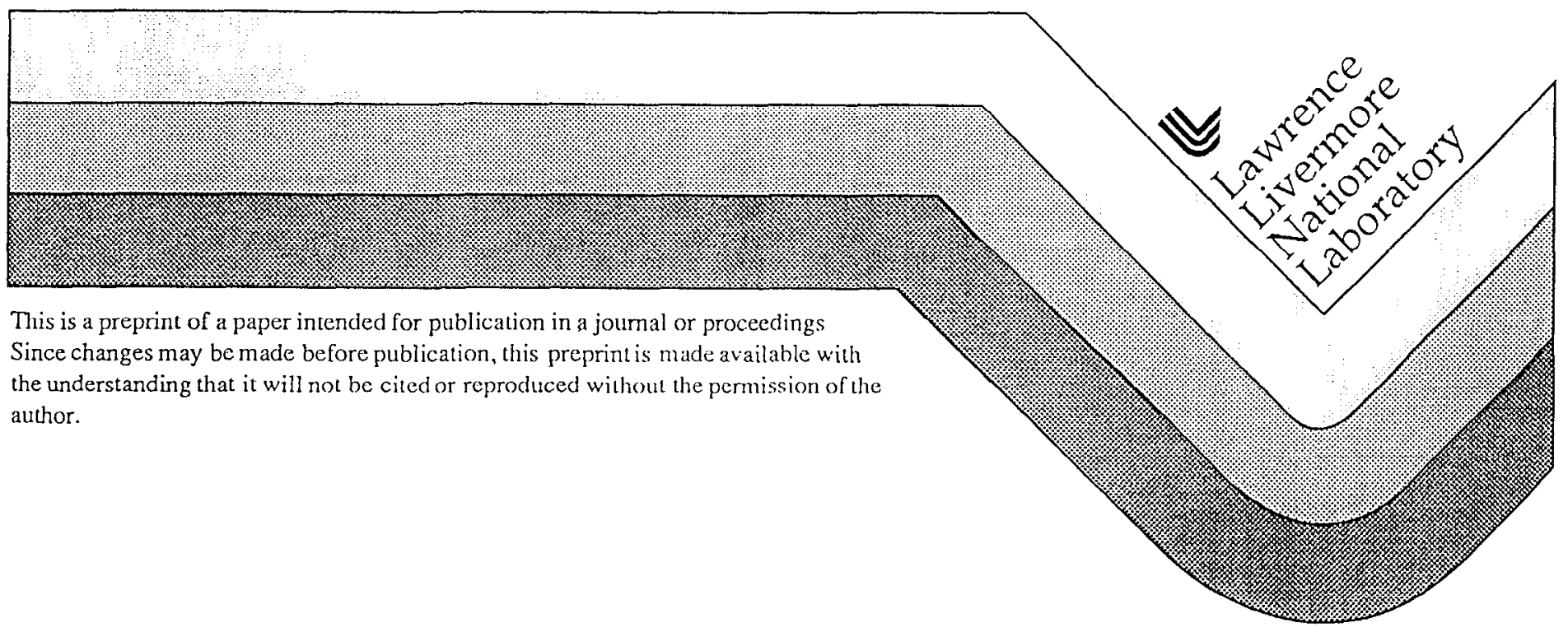




\section{DISCLAIMER}

This document was prepared as an account of work sponsored by an agency of the United States Government. Neither the United States Government nor the University of California nor any of their employees, makes any warranty, express or implied, or assumes any legal liability or responsibility for the accuracy, completeness, or usefulness of any information, apparatus, product, or process disclosed, or represents that its use would not infringe privately owned rights. Reference herein to any specific commercial product, process, or service by trade name, trademark, manufacturer, or otherwise, does not necessarily constitute or imply its endorsement, recommendation, or favoring by the United States Government or the University of California. The views and opinions of authors expressed herein do not necessarily state or reflect those of the United States Government or the University of California, and shall not be used for advertising or product endorsement purposes 


\section{Electromagnetic Interactions GEneRalized (EIGER): Algorithm Abstraction and HPC Implementation}

\author{
R. M. Sharpe, J. B. Grant, N. J. Champagne \\ Lawrence Livermore National Lab, Livermore, CA \\ D. R. Wilton, D. R. Jackson \\ University of Houston, Houston, TX \\ W. A. Johnson, R. E. Jorgensen \\ Sandia National Labs, Albuquerque, NM \\ J. W. Rockway, C. W. Manry \\ SPAWAR System Center, San Diego, CA
}

various shapes and dimensionality have appeared, as well as similar forms for bases representing the vector fields defined on these elements. These bases have robust computational propertics in both integral and partial differential equation formulations. Unified representations for mixed potential forms of Grcen's functions also exist, as do standard methods for handling their singular kernels. Combining thesc compact representations with object oriented software development methods means that it is now possible to develop very flexible general-purpose software for electromagnctic modcling. This paper describes one such computational environment currently under dcvclopment. The code, EIGER (Electromagnetic Interactions GEneRalized), will handle a variety of elcments---line scgments, triangles, quadrilaterals, tetrahedrals, prisms, and bricks---in both integral and partial differcntial equation formulations. The following sections describe different modeling features, which have been or will be included.

\section{GEOMETRY REPRESENTATION}

The EIGER physics kernel assumes that a geometrical description of a problem to be modeled is created by appropriate $\mathrm{CAD}$ mesh-generating software. The geometries from the mesh generator are combined with simulation specific information to produce an EIGER input file by a pre-processor that has data structures that are parallel to the physics kernel. The pre-processor can currently read a dozen different input formats for computation. The code suite uses a common representation for elements independent of dimensionality (2D or 3D) and order (linear, quadratic, etc.).

Convenient representations for the line segment, triangle, quadrilateral, tetrahedral, prism, and brick elements used in EIGER employ the Lagrange interpolation scheme 


$$
r=\sum_{i} r_{i} R_{i}(p, \xi)
$$

where $i=\left(i_{1}, i_{2}, \ldots, i_{n}\right)$ is a multi-index designating both the order and locations of interpolation points on curvilinear elements, and $\xi=\left(\xi_{1}, \xi_{2}, \ldots, \xi_{\mathrm{n}}\right)$ is a multi-vector of normalized coordinates defined on an element, one for each sub-boundary (endpoint, edge, or face of a line segment, surface, or volume element, respectively) comprising an element ${ }^{1} . R_{i}(p, \xi)$ is a Lagrange interpolation polynomial of order $p$ and has the separable form

$$
R_{i}(p, \xi)=R_{i_{2}}\left(p, \xi_{2}\right) \cdots R_{i_{n}}\left(p, \xi_{n}\right)
$$

where $R_{\mathrm{i}}(\mathrm{p}, \xi)$ is the Silvester-Lagrange interpolating polynomial $^{1,2}$. All additional geometrical quantities (e.g., element jacobian, edge vectors $l_{\mathrm{ij}}$, and coordinate gradient vectors $\nabla \xi$ ) may be obtained from the socalled unitary basis vectors $l_{\mathrm{i}}=\partial \mathrm{r} / \partial \xi_{\mathrm{i}}$ associated with the independent coordinates $\xi_{i}$. The detailed geometry of a 3D-prism element, depicting these quantities, is shown in Figure 1.

Careful examination of the prism element, along with the other elements of interest, clearly identifies information that all elements must have knowledge of. In EIGER, this information is cast into an element class (a fundamental class for geometry) which contains some of the following:

- An element type

- An element order

- A set of physical points that define the element

- The number of basis functions on the element

- Pointers to specific basis sets

- Additional attributes (possibly thickness or radius)

\section{OPERATORS}

The current development activity grew from research in integral equation methods. For dynamic problems, it is assumed that the unknowns associated with any element may be either equivalent electric or magnetic currents---or a combination of the two. Similarly, boundary conditions may involve either the electric field, the magnetic field, or both. Therefore electric and magnetic field operators of the following type are needed:

$$
\begin{gathered}
E(J, M)=-j \omega A(J)-\nabla \Phi(J) \\
-\frac{1}{\varepsilon} \nabla \times \boldsymbol{F}(\boldsymbol{M})
\end{gathered}
$$

$$
\begin{gathered}
H(J, M)=-j \omega F(M)-\nabla \Psi(M) \\
+\frac{1}{\varepsilon} \nabla \times A(J)
\end{gathered}
$$

These operators are expressed in terms of the electric and magnetic scalar potentials $\Phi$ and $\Psi$ and the magnetic and electric vector potentials $\boldsymbol{A}$ and $\boldsymbol{F}$ due to equivalent sources $J$ and $M$, respectively. The potential formulation minimizes the order of singularities that appear in the kernels of the associated integrodifferential operators. In order to completely determine the potentials, appropriate Green's functions, as discussed below, must also be specified.

In the current development both integral and partial differential equation formulations as well as hybrid formulations employing both types of operators are under way. Finite element method $\mathrm{s}$ directly attempt to solve partial differential equation formulations such as the vector Helmholtz equations

$$
\begin{aligned}
\nabla \times\left(\mu_{r}^{-1}\right. & \cdot \nabla \times E)-k_{0}^{2} \varepsilon_{r} \cdot \boldsymbol{E}= \\
& -j \omega \mu_{0} J-\nabla \times\left(\mu_{r}^{-1} \cdot M\right)
\end{aligned}
$$

or

$$
\begin{aligned}
\nabla \times\left(\varepsilon_{r}^{-1} \cdot \nabla \times \boldsymbol{H}\right)-k_{0}{ }^{2} \mu_{r} \cdot \boldsymbol{H}= \\
-j \omega \mu_{0} \boldsymbol{M}-\nabla \times\left(\varepsilon_{r}^{-1} \cdot J\right)
\end{aligned}
$$

The forcing functions are the source currents $J$ or $M$, which may be actual or equivalent sources.

Alternatively, the excitation may be due to sources outside a region's boundary. Both the differential and integral equation operators are enforced in a weak sense in order to minimize differentiability requirements on basis and testing functions.

Initially, EIGER was focused on frequency domain problems. However, the object-oriented structure of EIGER has facilitated extensions of the code to employ static operators. The unknown electric and magnetic currents ( $J$ and $M$ ) from the dynamic case are replaced by potentials and gradients of potentials respectively $(\Phi$ and $\partial \Phi / \partial \mathrm{n}$ ) otherwise the code structure remains identical. The code presently has the capability of modeling perfect electric conductors, perfect magnetic conductors, and dielectric materials both in 2 and 3 dimensions for static operators. Also, a hybrid FEM integral equation is available in $2 \mathrm{~d}$ and $3 \mathrm{~d}$. The EIGER pre-processor is being modified to output the associations needed for static analysis so once a structure is meshed it may be analyzed with either static or dynamic excitations.

and 


\section{BASES}

To compute the numerical solution of a problem and numerically enforce the chosen operator on the geometry, an appropriate set of expansion and testing functions is needed. The numerical advantages of using the Nedelec curl-conforming bases in the Helmholtz operators and the divergence-conforming bases in the integral operators are now well established ${ }^{3,4}$. Nedelec bases not only easily accommodate discontinuities in material properties, but also eliminate spurious modes using a minimum number of degrees of freedom per element for a given order of accuracy. Recently, high order interpolatory forms of the Nedelec bases have been constructed which are convenient as 'universal bases ' The unnormalized form of the divergenceconforming form of these bases of order $p$ is

$$
\Lambda_{i}^{\beta}(p, \xi)=\frac{\xi_{\beta}}{i_{\beta}} \hat{R}_{i}(p, \xi) \Lambda_{\beta}(\xi)
$$

where $\Lambda_{\beta}(\xi)$ is the usual (zeroth order) divergenceconforming basis associated with sub-boundary $\beta$ of an element and $R_{i}(p, \xi)$ is a modified Silvester-Lagrange polynomial similar to equation 2 but involving interpolation points shifted away from the element's boundaries.

Unnormalized curl-conforming bases have the form

$$
\Omega_{i}^{\beta}(p, \xi)=\frac{\xi_{\beta}}{i_{\beta}} \hat{R}_{i}(p, \xi) \Omega_{\beta}(\xi)
$$

for a set of bases associated with edge $\beta$ of a twodimensional element and

$$
\Omega_{i}^{\gamma \beta}(p, \xi)=\frac{\xi_{\gamma} \xi_{\beta}}{i_{\gamma} i_{\beta}} \hat{R}_{i}(p, \xi) \Omega_{\gamma \beta}(\xi)
$$

for a set associated with edges formed by the intersections of faces $\gamma$ and $\beta$ of a three-dimensional element. $\Omega_{\beta}$ and $\Omega_{\beta}$ are curl-conforming zeroth order bases associated with the elements.

When singular quantities such as the fields or currents near edges of conductors or dielectrics are modeled, higher order bases do not provide the expected increase in accuracy. To model such cases accurately, singular higher order bases are needed ${ }^{5}$. Such bases, as well as special basis functions for modeling junctions between surfaces and wires, are incorporated into EIGER ${ }^{6}$.
GREEN'S FUNCTIONS

For efficient integral equation solution capabilities, a number of Green's function capabilities are desired. Both two- and three-dimensional Green's functions and their gradients are available in EIGER. A wide variety of problem types may be handled if multi-layered media Green's functions for both periodic and nonperiodic media are available. The mixed potential integral equation (MPIE) formulation ${ }^{7}$ for such problems is particularly convenient in practical computations. A typical potential in (3), say the magnetic vector potential, is expressed as an integral over sources $J$ on a domain $D$ as

$$
A=\int_{D} G^{A}\left(r, r^{\prime}\right) \cdot J\left(r^{\prime}\right) d D
$$

The Green's potential dyad, $G^{A}$, may in turn be written as

$$
\begin{aligned}
G^{A}\left(\boldsymbol{r}, \boldsymbol{r}^{\prime}\right)=I G_{0}\left(\boldsymbol{r}, \boldsymbol{r}^{\prime}\right) & +\sum_{i} \Gamma_{i} G_{0}\left(\boldsymbol{r}, \boldsymbol{r}_{i}^{\prime}\right) \\
& +\Delta G^{A}\left(\boldsymbol{r}, \boldsymbol{r}^{\prime}\right)
\end{aligned}
$$

where $I$ is the identity dyad, $G_{0}$ is the background homogeneous medium Green's function for nonperiodic media, $\Gamma_{\mathrm{i}}$ is a dyadic reflection coefficient representing a quasi-static image located at $r_{\mathrm{i}}^{\prime}$, and $\Delta G^{\mathrm{A}}\left(r, r^{\prime}\right)$ is a relatively smooth integral contribution of Sommerfeld type. The latter integral is efficiently evaluated using a combination of complex path deformation and the method of averages ${ }^{8}$. For periodic media, $G_{0}$ is the homogeneous media periodic Green's function, an infinite series that may be efficiently evaluated using the Ewald method ${ }^{9}$. In this case $\Delta G^{A}$ is also a rapidly converging series: For complete generality, it is possible to separately model the environment on either side of a surface element using any Green's function available to the code.

Other important Green's functions for applications are those that may be constructed using reflection or rotational symmetries. These symmetry operators may be constructed by appropriately reflecting or rotating source elements and endowing them with appropriate signs or phase factors.

\section{ELEMENT MATRIX CONSTRUCTION}

The first step in obtaining a matrix approximation to an operator equation is to form the element matrix. This 
involves all interactions between basis functions defined on each pair of elements in the integral equation case, or within each individual element in the differential equation case. This difference between the two cases accounting for the sparcity of the system matrix associated with the FEM approach. Assuming that the testing functions are the same as the bases, typical entries in the element matrices for integral equations are

$Z_{i j}^{\gamma \beta}=j \omega L_{i j}^{\gamma \beta}+\frac{1}{j \omega} S_{i j}^{\gamma \beta}$

where

$L_{i j}^{\gamma \beta}=\left\langle\Lambda_{i}^{\gamma} ; G^{A} ; \Lambda_{j}^{\beta}\right\rangle$,

$S_{i j}^{\gamma \beta}=\left\langle\nabla \cdot A_{i}^{\gamma} ; K^{\Phi} ; \nabla \cdot A_{j}^{\beta}\right\rangle$

used when the electric field due to electric current sources is required. Its dual, the operator corresponding to the magnetic field due to magnetic current sources, used in aperture or dielectric problems, has the form

$Y_{i j}^{\gamma \beta}=j \omega C_{i j}^{\gamma \beta}+\frac{1}{j \omega} \Gamma_{i j}^{\gamma \beta}$

where $C_{i j}{ }^{\beta}$ and $\Gamma_{i j}{ }^{\beta}$ are also defined by duality. Similar representations exist corresponding to electric fields due to magnetic currents and their dual, magnetic fields due to electric currents.

In three dimensions, the element matrix entries for the electric field form of the Helmholtz operator (5) are

$Y_{i j}^{\alpha \beta \gamma \delta}=j \omega C_{i j}^{\alpha \beta \gamma \delta}+\frac{1}{j \omega} \Gamma_{i j}^{\alpha \beta \gamma \delta}$

where

$C_{i j}^{\alpha \beta \gamma \delta}=\varepsilon_{0}\left\langle\Omega_{i}^{\alpha \beta} ; \varepsilon_{r} ; \Omega_{j}^{\gamma \delta}\right\rangle$,

$\Gamma_{i j}^{\alpha \beta \gamma \delta_{-}}=\mu_{0}^{-1}\left\langle\nabla \times \Omega_{i}^{\alpha \beta} ; \mu_{r}^{-1} ; \nabla \times \Omega_{j}^{\gamma \delta}\right\rangle$

A corresponding dual form exists for the magnetic form of the Helmholtz operator ${ }^{6}$.

The generalized form of the inner product notation used above allows for the appearance of dyadic quantities in the inner products; when these dyads are also potential quantities, an extra integration over source coordinates is also implied. The similarity in form of the element matrices for both integral and partial differential equations allows similar algorithms to be used. The construction of these element matrices is essentially the core of any computational engine, and nearly all of the possible combinations above have been implemented in EIGER. A key feature in evaluating the singular integrals which appear is the existence of closed formulas for the quasi-static contribution of various potentials for constant and linear source densities on polygonal and polyhedral domains ${ }^{10}$.

\section{QUADRATURE}

A variety of quadrature schemes must be available for use in a general-purpose code. These include:

- One-dimensional Gaussian quadrature rules for various orders and types of singular integrands.

- Various order Gaussian quadrature rules for triangles; for quadrilaterals, mappings can be made to forms such that cartesian product quadrature rules can be constructed.

- Various order Gaussian quadrature rules for tetrahedrons; for prism and bricks, mappings may be made to forms for which cartesian product rules may be used.

- Various special purpose schemes such as adaptive integration.

Tables corresponding to various quadrature schemes are stored as a module in EIGER and pointers are used to select the appropriate coefficients and weights for element matrix evaluation.

\section{EXCITATIONS}

Problems such as the determination of dispersion on guided wave structures, cutoff frequencies for waveguides, or resonant frequencies of cavities do not require excitation sources. If the system matrix for these source-free problems is linear with respect to the quantity of interest, then it may be determined by eigenvalue solution; if not, it is determined by searching for roots of the determinantal equation.

Radiation, scattering, and penetration problems are not source-free and may be distinguished primarily by the location of excitation. Antenna or radiation problems generally are excited by near field sources such as delta-gap or frill sources. 'The weak forms used in EIGER permit these quantities to be expressed simply as voltages across terminal pairs. In scattering and penetration problems plane wave excitations are needed. An important point to observe is that once a variety of Green's functions are available for 
constructing element matrices, these can also be used to construct excitation by near field sources. Equally important in reducing post-processing software is using reciprocity to determine the fields radiated by equivalent currents. That is, the field at a point in space may be determined using the matrix excitation vector for a point source located at that point.

\section{BOUNDARY CONDITIONS}

EIGER can handle a variety of boundary conditions and is constructed so that new ones may be added as needed. As currently implemented, the electric field (EFIE), magnetic field (MFIE), or combined field (CFIE) integral equations may be used on conducting surfaces. For aperture problems, the aperture electric field may be determined in terms of equivalent magnetic currents. The continuity conditions of electric and magnetic fields are used at dielectric interfaces to determine equivalent electric and magnetic currents there. Equivalent currents may be assumed to exist on either side of a surface element. Thus for open conductors, it is possible to close the conductor by aperture surfaces and determine not only the aperture fields, but also the separate surface currents on the exterior and interior conducting surfaces.

Both lumped and distributed impedance loading of elements is permitted in EIGER through a simple impedance boundary condition, and extensions to more general boundary conditions, such as shells and coatings, are currently in progress. Also, the variety of boundary conditions for static solutions was discussed earlier.

\section{GLOBAL MATRIX ASSEMBLER}

Clearly, elements of the element matrix correspond to pairs of global unknown (degree of freedom) indices, which in turn correspond to storage locations in the system matrix. It is the job of the matrix assembler to determine how element matrix contributions are to be stored in the global system matrix. A number of further index mappings may be needed in addition to those just described, however. For example, an additional index mapping may be needed to employ a sparse matrix storage scheme for matrices generated by partial differential equation formulations. Additional mappings may be needed to store elements in certain blocks for partitioned matrix solutions or for mapping to different multi-processors. Other mappings may be needed to account for an object's symmetry, or to utilize special formulations at low frequencies to eliminate matrix instabilities. Many of these mappings are currently available in EIGER.

\section{LINEAR SYSTEM SOLVERS}

EIGER currently uses standard LINPACK routines for the direct solution of the dense, complex matrices arising in integral equation procedures on a serial platform. A complex-symmetric matrix solution algorithm may be chosen if appropriate. A conjugategradient solution algorithm is also available and more sophisticated iterative solvers are to be added. Because many robust algorithms are widely available, and because machine-specific solvers may be needed in multi-processor environments, concentration has not focused on development of solvers for EIGER.

It is anticipated that sparse matrix and eigenvalue solvers will be needed for partial differential equation formulations. Special purpose solvers may also be needed for the block sparse matrices arising in hybrid formulations.

\section{HPC IMPLEMENTATION}

During the design of the EIGER software architecture many different issues were addressed. One of these concerned the computer platforms that the code would be well tuned for. A decision was made early to not limit target platforms. This was addressed by identifying the flexibility needed for different architectures (single processor, multi-processor, shared memory, distributed memory, etc.) and addressing these issues during initial design. This yields a package that is not encumbered by legacy software issues when trying to port to different platforms.

The initial port to parallel platforms was for distributed memory (MIMD) architectures (DEC Alpha clusters and IBM SP2). For these machines, the linear algebra solvers usually dictate the manner in which the algorithm is to be distributed. The primary concerns here are bandwidth and latency issues.

Since the solution of the present set of operators yields a linear system of equations, a given problem is partitioned based upon the matrix equations (not based upon the geometry directly). The present parallel solution algorithms employ a block matrix partitioning scheme, which is then used to distribute the clectromagnetic calculations at run time.

Future HPC considerations will address shared memory (threaded) algorithms and hybrid parallel algorithms. 


\section{SUMMARY}

It is possible to develop an efficient general-purpose electromagnetic solver primarily because of a combination of relatively recent computational and technological developments:

- Development of a convenient and unified indexing and representation scheme for interpolatory basis functions of arbitrary order on any of the canonical element shapes.

- Development of robust representations and means for computing Green's functions for both periodic and nonperiodic, multi-layered media.

- Development of a unified approach for handling Green's functions singularities.

- Ability of advanced languages to create complex data types like vectors, dyads, and even more complex objects, as well as to create operators, such as dot and cross products, which can operate on them.

- Capability of advanced languages to dynamically dimension arrays, which allows, for example, efficient handling of arrays of variable size when selecting or mixing various element shapes, orders of geometrical or unknown representation, orders of quadrature, or boundary conditions.

- Organization of the computational paradigm into an object-oriented approach by abstracting algorithms, encapsulating data, giving inheritance to data objects, and developing code in modular form.

Careful abstraction and generalization of each step in the numerical algorithm yields a code, which is maintained easily and allows for future expansion.

\section{ACKNOWLEDGMENTS}

This work was performed under the auspices of the US Department of Energy by Lawrence Livermore National Laboratory under contract No. W-7405-Eng48 and by Sandia National Laboratories, a inuliprogram laboratory operated by Sandia Corporation, a Lockheed Martin Company, under contract No. DE-AC04-94AL85000.

\section{BIBLIOGRAPHY}

[1] R. D. Graglia, D. R. Wilton, and A. F. Peterson, "Higher Order Interpolatory Bases for Computational
Electromagnetics," IEEE Trans. Antennas Propagat., vol. AP-45, March 1997, pp. 329--342.

[2] P. P. Silvester and R. L. Ferrari, Finite Elements for Electrical Engineers. Cambridge: Cambridge Press, 1990.

[3] A. F. Peterson and D. R. Wilton, "Curlconforming mixed-order edge elemetns for discretizing the 2-D and 3-D vector Helmholtz equation," in Finite Element Software for Microwave Engineering. T. Itoh, G. Pelosi, and P. P. Silvester, Eds. New York: Wiley, 1996, pp. 101--125.

[4] D. Sun, J. Manges, X. Yuan and Z. Cendes, "Spurious modes in finite element methods," IEEE Antennas Propagat. Mag., vol. 37, no. 5, pp. 12--24, Oct. 1995.

[5] W. J. Brown, "Higher Order Modeling of Surface Integral Equations," Ph.D. Dissertation, Univ Houston, Dec., 1996.

[6] S.-U. Hwu, "'Electromagnetic Modeling of Conducting and Dielectric Coated Wire, Surface, and Junction Configurations" Ph.D. dissertation, Univ. Houston, Houston, TX, May 1990.

[7] K. A. Michalski and D. Zheng, "Electromagnetic scattering and radiation by surfaces of arbitrary shape in layered media, Part I: Theory," IEEE Trans. Antennas Propagat., vol. AP-38, March 1990, pp. 335--344.

[8]J. Mosig, "Integral Equation Technique", Chapt. 3 in T. Itoh, ed., Numerical Techniques for Microwave and Millimeter-Wave Passive Structures, Wiley, NY, 1989.

[9] K. E. Jordan, “An efficient numerical evaluation of the Green's function for the Helmholtz operator on periodic structures," J. Comp. Phys., vol. 63, no. 1, March 1986, pp. 222-- 235.

[10] D. R. Wilton, S. M. Rao, A. W. Glisson, D. H. Schaubert, O. M. Al-Bundak, and C. M. Butler, "Potential integrals for uniform and linear source distributions on polygonal and polyhedral Domains," IEEE Trans. Antennas Propagat., vol. AP-32, March 1984, pp. 276--281. 


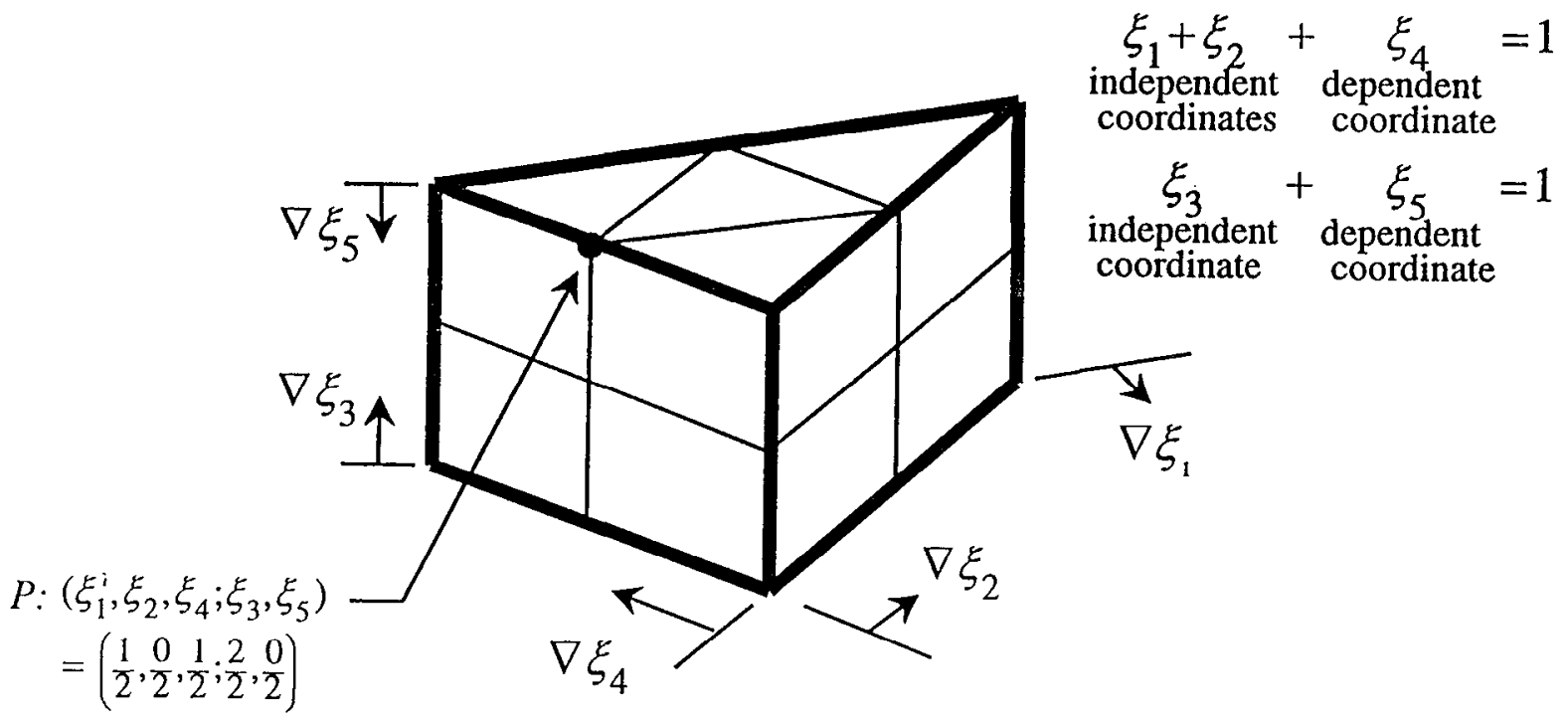

coordinate multivector of point P: $\quad \xi=\left(\xi_{1}, \xi_{2}, \xi_{4} ; \xi_{3}, \xi_{5}\right)=\left(\frac{1}{2}, \frac{0}{2}, \frac{1}{2} ; \frac{2}{2}, \frac{0}{2}\right)$

multi-index of point P: $\quad i=\left(i_{1}, i_{2}, i_{4} ; i_{3}, i_{5}\right)=(1,0,1 ; 2,0)$

Figure 1. Index and coordinate system notation for the prism element of order $q=2$. 



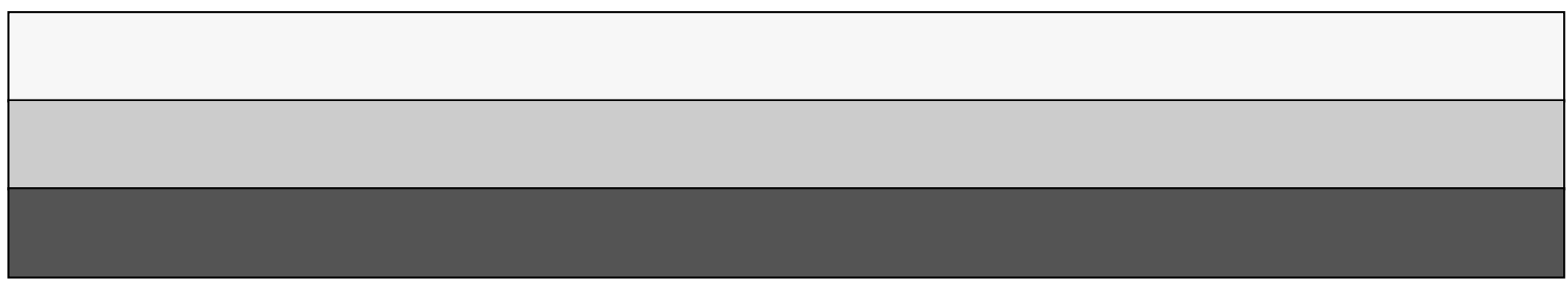

